\title{
Halogen bonds and other noncovalent interactions in the crystal structures of 1,2-diiodo alkenes: an ab initio and QTAIM study
}

\author{
Yongna Yuan ${ }^{1}$, Matthew Mills ${ }^{2}$, Fuyang $\mathrm{Li}^{1}$, Yuhong $\mathrm{Du}^{1}$, Jiaxuan $\mathrm{Wei}^{1}$, and Wei $\mathrm{Su}^{1}$ \\ ${ }^{1}$ Lanzhou University \\ ${ }^{2}$ Joint BioEnergy Institute
}

July 29, 2020

\begin{abstract}
A series of interatomic interactions interpretable as halogen bonds involving I... I, I... O, and I. . C ( $\pi$ ), as well as the noncovalent interactions I...H and O... O were observed in the crystal structures of 1,2-diiodoolefins dimers according to ab initio calculations and the quantum theory of "atoms in molecules" (QTAIM) method. The interplay between each type of halogen bond and other noncovalent interactions was studied systematically in terms of bond length, electrostatic potential and interaction energy, which are calculated via ab initio methods at the B3LYP-D3/6-311++G(d,p) and B3LYP-D3/def2-TZVP levels of theory. Characteristics and nature of the haologen bonds and other noncovalent interactions, including the topological properties of the electron density, the charge transfer and their strengthening or weakening, were analyzed by means of both QTAIM and "natural bond order" (NBO). These computational methods provide additional insight into observed intermolecular interactions and are utilized to explain the differences seen in the crystal structures.
\end{abstract}

\section{KEYWORDS}

Halogen bonding; noncovalent interaction; ab initio calculation; Quantum theory of atoms in molecules; topological properties

\section{1 | INTRODUCTION}

Halogen bonds have recently been extensively studied as they play an essential role in medicinal chemistry ${ }^{1}$, molecular recognition ${ }^{2}$, material science ${ }^{3}$, and crystal engineering ${ }^{4,}{ }^{5}$. Halogen bonds are weak, noncovalent interactions between an electrophilic region of a halogen and a nucleophile ${ }^{6}$. Halogen bonds can schematically be depicted $\mathrm{R}-\mathrm{X}[$ ? ] Y, where $\mathrm{X}$ is a halogen (typically $\mathrm{I}, \mathrm{Br}, \mathrm{Cl}$, and rarely $\mathrm{F}$ ), and $\mathrm{Y}$ is defined as the halogen bond donor ${ }^{7,8}$. X acts as an electron acceptor for the interaction, whereas $\mathrm{Y}$ is typically an electron rich $\mathrm{O}, \mathrm{N}, \mathrm{S}$, or $\mathrm{Y}$-donor functional group (e.g. $\pi$-electron systems or aromatic surfaces). A halogen atom may be covalently bound to one or several atoms and can additionally form one or several halogen bonds simultaneously ${ }^{6,9}$.

Halogen bonds have similarities with hydrogen bonds and involve the same mechanisms ${ }^{10}$. In 2008, Metrangolo et al. summarized the similarities and differences between halogen bonding and hydrogen bonding complexes $^{4}$. Kirk et al. investigated the competition between hydrogen bonding and halogen bonding for the $(\mathrm{Y}=\mathrm{Cl}, \mathrm{Br}, \mathrm{I}, \mathrm{At}) /$ halogenabenzene $/ \mathrm{NH}_{3}$ systems and concluded that hydrogen bonding has an advantage when the halogen is $\mathrm{Cl}$, while halogen bonds tend to be formed when the halogen atom $\mathrm{Y}=\mathrm{I}^{11}$. Halogen bonds have been observed in crystal structures containing halogen atoms ${ }^{12}$. Due to their geometric properties, halogen bonds are considered efficient tools in designing the structures of crystals ${ }^{4,13}$. The concept of halogen bonds in crystal engineering attracts increasing attention as they can be pivotal in the stability of crystals $^{14,15}$. 
Although halogen bonding was first observed two centuries $\operatorname{ago}^{7}$, the fundamentals of its nature and its potential applications in crystal engineering have remained unexplored until recently. In most cases reported in the literature, halogen bonds were studied in co-crystals between two different compounds, one of which being the halogen bond donor and the other is the acceptor ${ }^{12,16}$. On the basis of crystallographic and computational studies, this type of interaction was shown to predominantly originate from charge transfer and electrostatics. Later, the interaction was also found to possess polarization and dispersion contributions. In 2014, Deepa et al. carried out a theoretical study of a series of organic crystal structures containing various halogen bonds and found the strongest halogen bonds involed iodine as both halogen-bond donor and acceptor ${ }^{17}$. In the following year, Koskinen et al. carried out a detailed study of unexpectedly strong $\mathrm{I}^{+} \ldots \mathrm{S}$ halogen bonds in $\left[\mathrm{I}(2 \text {-imidazolidinethione })_{2}\right]^{+}$with the results supporting the coordinative nature of the halogen bond ${ }^{18}$.

Furthermore, topological properties, vibrational frequencies, interaction energies, and charge transfer in halogen-bond-containing systems have been studied using both Bader's quantum theory of atoms in molecules (QTAIM) $)^{19}$ and Weinhold's "natural bond orbital NBO" methods. A variety of important concepts, such as critical points, bond path, gradient path of electron density, and the Laplacian of electron density are generated through QTAIM theory. Clark et al. calculated the electrostatic potential of the series of molecules $\mathrm{CF}_{3} \mathrm{X}(\mathrm{X}=\mathrm{F}, \mathrm{Cl}, \mathrm{Br}$ and $\mathrm{I})$ and found that the three unshared pairs of electrons produced a belt of negative electrostatic potential around the central region of the $\mathrm{X}$ atom (except for $\mathrm{F}$ ), leaving the outermost region positive, which designated the " $\sigma$-hole" ${ }^{20}$. They also discovered that the strength of the $\sigma$-hole depends on the nature of the halogen atom. The more polarizable and the lower the electronegativity of the halogen atom, the more positive the $\sigma$-hole. Thus, the interaction strength of halogen bonds increases in the order of $\mathrm{F}<\mathrm{Cl}<\mathrm{Br}<\mathrm{I}$. It is the $\sigma$-hole that allows the halogen atom to form a halogen bond with a Lewis base $^{21}$ and that makes the A... X-R angle tend towards a linear configuration ${ }^{10}$. In 2010, Zeng et al. comparatively analyzed the properties of halogen bonds and hydrogen bonds by QTAIM calculations and concluded that the two interactions were coincident in topological properties ${ }^{22}$. In the following year they analyzed halogen bonds between sulfides and dihalogen molecules and found that electrostatic interactions played an important role in these halogen bonds ${ }^{23}$. Grabowski et al. calculated the QTAIM characteristics of halogen bonds, dihalogen bonds and halogen-hydride bonds ${ }^{24}$. In 2018, Bauzá et al. analyzed the interplay between $\pi$-hole and lone pair[?] $\pi / \mathrm{X}-\mathrm{H}[?] \pi$ interacitons through QTAIM calculations ${ }^{25}$. In 2019, Benito et al. first reported the cocrystallization of an adenine derivative which acts as a halogen bond acceptor; the calculations were carried out via DFT calculations and the QTAIM method ${ }^{26}$. In 2020, Wzgarda-Raj et al. investigated several observed types of halogen bonding interactions in a series of cocrystals in detail based on QTAIM ${ }^{27}$. In addition to the traditional halogen bonds, Domagala et al. built a model of a double $(+/-$ ) charge-assisted halogen bridge for a set of quinuclidine-like cation derivatives and anions, these charged fragments were observed to form strong halogen bonding complexes, with interaction energies high as 100 $\mathrm{kcal} / \mathrm{mol}^{28}$.

In this work, calculations carried out on new crystal structures of 1,2-diiodoolefines are reported, including nine monomers and nine dimers. These crystal structures were previously synthesised by Hettstedt et al . in $2015^{29}$ (see Figure 1). The purpose of this study is to investigate the characteristics and properties of halogen bonds (i.e. I...I, I... O, and I...C $(\pi)$, where $\mathrm{C}(\pi)$ can be aromatic, aliphatic or acetylenic $\pi$-systems) and other noncovalent interactions such as I...H and O... O observed in these crystal structures.

\section{FIGURE 1 is near here}

\section{2 | COMPUTER METHOD}

Data for halogen bonds observed in crystal structures of 1,2-diiodoolefins reported by Hettstedt et al. ${ }^{29}$ have been used as references for quantum chemical calculations to analyze noncovalent interactions. The structures of monomers and dimers were obtained from the Cambridge Crystal Structure Database (CCSD). Geometry optimization, molecular electrostatic potential, and interaction energy calculations were carried out using the Gaussian09 program package ${ }^{30-33}$. The DFT-D3 method, which is recommended in studying noncovalent interactions ${ }^{34-37}$, was applied to the monomer and dimer optimizations. Both Kolar et al. and 
Banza et al. ${ }^{38,} 39$ verified that the B3LYP-D3 method combined with the "def2" basis set series can be used to successfully examine halogen bonds and the properties of $\sigma$-holes. Therefore, the B3LYP-D3/6-311++G(d,p) and B3LYP-D3/def2-TZVP levels of theory were used to optimize the structures of monomers. Frequency calculations were run to be sure that the geometry was a potential energy minimum (no negative frequencies were obtained). The keyword "counterpoise" was used for the calculations of corrected interaction energies $([?] E(\mathrm{AB}))$ including the inherent basis set superpositon error (BSSE) ${ }^{40}$ according to Eq. (1).

$$
\text { [?] } E(\mathrm{AB})=E(\mathrm{~A}, \mathrm{~B})-\{E(\mathrm{~A})+E(\mathrm{~B})\}+\operatorname{BSSE}(1)
$$

Here, $E(\mathrm{AB})$ is the total energy of dimer $\mathrm{AB}$ and $E(\mathrm{~A})$ and $E(\mathrm{~B})$ are the energies of monomers $\mathrm{A}$ and $\mathrm{B}$, respectively.

The electrostatic potential on the molecular surfaces of all monomers was analyzed in order to gain insight into the nature and directionality of the halogen bond interactions being considered herein. The electrostatic potentials were considered to be an outer contour of the electron density, and were cut off at the 0.001 au (electrons/bohr ${ }^{-3}$ ) surface, as proposed by Bader et al. ${ }^{41}$. The most positive value of the potentials (the local maximum) is referred to as $\mathrm{V}_{\mathrm{S} \text {,max }}$. Natural bond orbital (NBO) calculations were performed using the NBO 3.1 program $^{42}$ as implemented in Gaussian09. The QTAIM theory was applied to find critical points and these were analyzed in terms of electron density and the Laplacian. The topological properties at the bond critical points (BCPs) of halogen bonds were computed with the program-AIMALL $2000^{43}$.

\section{3 | RESULT AND DISSCUSSION}

\section{1 | Monomers}

\subsection{1 | Geometries}

In the work reported in ref. [29], trans isomers were obtained for all systems but one: acetal 7, for which a mixture of cis (7a) and trans (7b) derivatives were formed in the synthetic process. Therefore, there are in total nine monomers reported on in this study. All of their geometries were optimized at the B3LYPD3/6-311++G(d,p) and B3LYP-D3/def2-TZVP levels of theory. Figure 2 shows the nine 1,2-diiodoolefin structures optimized at the B3LYP-D3/6-311++G(d,p) level of theory. The $\mathrm{C}=\mathrm{C}$ bond lengths (the common part in the nine 1,2-diiodoolefin structures) in both the optimized and crystal structures are listed in Table 1. It can be seen that the bond lengths of the $\mathrm{C}=\mathrm{C}$ in the nine monomers calculated at the B3LYP-D3/6$311++\mathrm{G}(\mathrm{d}, \mathrm{p})$ and B3LYP-D3/def2-TZVP levels of theory are all in good agreement with the values seen in the crystal structures. Also, there is only a marginal difference between the $\mathrm{C}=\mathrm{C}$ bond lengths optimized at the two levels of theory. Therefore, considering the computational cost, the B3LYP-D3/6-311++G(d,p) level of theory was applied to carry out all molecular electrostatic potential (Vs,max) calculations.

FIGURE 2 is near here

TABLE 1 is near here

\subsection{2 | Electrostatic Potentials}

Fig. 3 shows contour maps of the electrostatic potential for monomers 1-8. The $\sigma$-hole of the two iodine

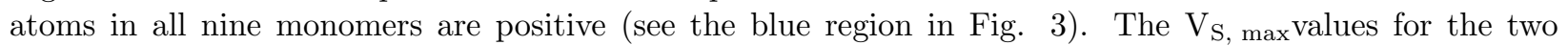

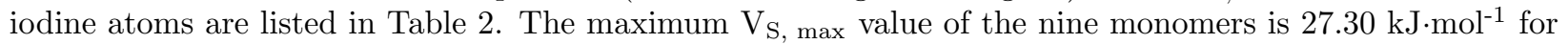
monomer 8. Monomers 5 and 6 had the second and third largest $\mathrm{V}_{\mathrm{S} \text {, max }}$ values of $22.87 \mathrm{~kJ} \cdot \mathrm{mol}^{-1}$ and 22.32 $\mathrm{kJ} \cdot \mathrm{mol}^{-1}$ respectively. The $\mathrm{V}_{\mathrm{S}}$ max values for monomers $7(\mathrm{a})$ and $7(\mathrm{~b})$ are the smallest among all monomers, which are $14.96 \mathrm{~kJ} \cdot \mathrm{mol}^{-1}$ and $15.18 \mathrm{~kJ} \cdot \mathrm{mol}^{-1}$, respectively. Based on a comparison of all the studied monomers, the iodine atom's chemical environment most strongly affects the electrostatic potential of iodine's $\sigma$-hole.

FIGURE 3 is near here

TABLE 2 is near here

\section{2 | Dimer}




\subsection{1 | Geometries}

Six 6 dimers of diiodoalkene were provided in the experimental supplementary data of ref. [29], including dimers $2,3,5,6,7 \mathrm{~b}$ and 8 . Each of these dimer structures was partially geometry optimized at the B3LYPD3/6-311++G(d,p) and B3LYP-D3/def2-TZVP levels of theory. Because the dimers were too large to be

fully geometry optimized, partial atoms or groups were fixed to ensure optimization is successful, with the fixed atoms or groups chosen to be far away from the locationsof the noncovalent bonds (e.g. I...I, I... O, I... C $(\pi), \mathrm{I} \ldots \mathrm{H}$ and $\mathrm{O} \ldots \mathrm{O}$ ) formed. The details of fixed atoms or groups for each dimer are listed in the Supporting Information. The geometries optimized at the B3LYP-D3/6-311++G(d,p) level of theory are show in Figure 4. The noncovalent bond lengths of each dimer, including the values in crystal structures and the values calculated at the B3LYP-D3/6-311++G(d,p) and B3LYP-D3/def2-TZVP levels of theory, are listed in Table 3. The deviations between the crystal structure values and the values calculated at the two levels of theory are quite small. The average deviation between the noncovalent bond lengths optimized at the B3LYP-D3/6-311++G(d,p) level of theory and the values in crystal structures are $0.123 \AA$, and the corresponding deviation is $0.101 \AA$ between the values optimized at the B3LYP-D3/def2-TZVP levels of theory and the crystal structure values. Again, the noncovalent bond lengths optimized at these two levels of theory are similar. Because the electron charge distribution of the halogen atom is anisotropic, the halogen can act both as the Lewis acid and as the Lewis base ${ }^{44-47}$. This is why dihalogen bonds are possible in the 6 dimers.

\section{FIGURE 4 is near here}

\section{TABLE 3 is near here}

\subsection{2 | Interaction Energies}

There are five different types of intermolecular noncovalent interactions in the six diiodoalkene dimers (see Figure 4): I. . I, I. . . O, I. . C ( $\pi)$, I. . H and O. . O. Interaction energy is an importantmeasure of the strength of an intermolecular interaction. The interaction energy in the dimers can be regarded as the energy difference between the dimer and the monomers as captured via Eq. (1). Table 4 summarizes the interaction energies of the halogen bonds in the six dimers. All results were corrected for BSSE by using counterpoise methods. Naturally, the effect of BSSE correction is prominent for halogen bonds. Calculations are carried out for crystal and optimized dimers, respectively, to explore whether if it is necessary to optimize the crystal structure geometries.

\section{TABLE 4 is near here}

Of all the dimers shown in Figure 4, dimer 2 possesses the smallest number of intermolecular noncovalent bonds: two halogen bonds of I... I and I.... H. Its interaction energies calculated at the B3LYP-D3/6$311++\mathrm{G}(\mathrm{d}, \mathrm{p})$ level of theory are $-9.58 \mathrm{~kJ} \cdot \mathrm{mol}^{-1}$ and $-11.67 \mathrm{~kJ} \cdot \mathrm{mol}^{-1}$ respectively for crystal and optimized geometries; and the respective values are $-11.7 \mathrm{~kJ} \cdot \mathrm{mol}^{-1}$ and $-12.57 \mathrm{~kJ} \cdot \mathrm{mol}^{-1}$ at the B3LYP-D3/def2-TZVP level of theory. Dimer 3 contains three halogen bonds of I. . I, I. . C $(\pi)$, I $2 \ldots$ C $27(\pi)$, and I17 . C12 $(\pi)$. Dimer 3 has the second highest interaction energy (Table 4) due to the two strong I...C( $\pi$ ) noncovalent bonds. Similarly, the I. . C $(\pi)$ noncovalent bond was also found in dimers 5 and 6 ; I23. . C11 $(\pi)$ in dimer 5 and I1 . . C 33 $(\pi)$ in dimer 6 . Moreover, the halogen bond of type I. . O was found in dimers 5 (I2 . . O25) and 6 (I27... O3). The respective interaction energies computed at the two levels of theory are $-29.44 \mathrm{~kJ} \cdot \mathrm{mol}^{-1}$ and $-32.02 \mathrm{~kJ} \cdot \mathrm{mol}^{-1}$ for crystal dimer 5 and $-34.19 \mathrm{~kJ} \cdot \mathrm{mol}^{-1}$ and $-31.79 \mathrm{~kJ} \cdot \mathrm{mol}^{-1}$ for the geometry optimized dimer 5 . Equally, the respective interaction energies computed at the two levels of theory are $-24.59 \mathrm{~kJ} \cdot \mathrm{mol}^{-1}$ and $-26.45 \mathrm{~kJ} \cdot \mathrm{mol}^{-1}$ for crystal dimer 6 and $-29.86 \mathrm{~kJ} \cdot \mathrm{mol}^{-1}$ and $-27.00 \mathrm{~kJ} \cdot \mathrm{mol}^{-1}$ for the optimized dimer. The interaction energy in dimer $7 \mathrm{~b}$ is also very high because there are five halogen bonds in dimer $7 \mathrm{~b}$ : one I...I, one I... C $(\pi)$ and three I...H, the details are shown in Figure 4 and Table 4. The highest interaction energy occurs in dimer 8 , and the corresponding interaction energies computed at the two levels of theory are $-36.75 \mathrm{~kJ} \cdot \mathrm{mol}^{-1}$ and $-39.09 \mathrm{~kJ} \cdot \mathrm{mol}^{-1}$, respectively, for crystal dimer 8 , and are $-44.55 \mathrm{~kJ} \cdot \mathrm{mol}^{-1}$ and -39.95 $\mathrm{kJ} \cdot \mathrm{mol}^{-1}$ for optimized dimer 8 . This Dimer has three halogen bonds of I... O, I...C $(\pi)$ and I...H and one O... O noncovalent bond. To summarize, in Table 4, both the interaction energies (E_Int) and the BSSE 
energies (E_BSSE) for crystal dimers calculated at the two levels of theory are very close to the values for the optimized dimers. Therefore, the properties of halogen bonds can be calculated directly using the crystal structures without geometry optimization.

\subsection{3 | Natural Bond Orbital Analysis}

To better understand the intermolecular interactions, natural bond orbital (NBO) analysis was carried out to characterize the weak interactions. Formation of complexes involing noncovalent bonds is associated with an orbital interaction between the bonding orbital in the electron donor and the antibonding orbital in the electron acceptor. Table 5 lists the second-order perturbation energy $\left(E^{(2)}\right)$ and the charge transfer $([?] q)$ obtained by $\mathrm{NBO}$ analysis. Both $E^{(2)}$ and [?] $q$ represent the transfer from one molecule (donor) to the other molecule (acceptor) in the six dimers. Owing to the time-consuming nature of the B3LYP-D3/def2-TZVP level of theory, all NBO calculations were carried out at the B3LYP-D3/6-311++G(d,p) level of theory. The second-order perturbation energy represents the degree of charge transfer from the bonding orbital to the antibonding orbital, which is the degree of electron delocalization. Ultimately, the second-order perturbation energy allows us to quantitatively evaluate the charge transfer due to the formation of the halogen bond.

The results listed in Table 5 show that there is a positive relationship between the second-order perturbation energy $E^{(2)}$ and the charge transfer [?]q in the studied systems. Due to the centrosymmetry of dimer 3 , the charge transfer from one monomer to another in both the crystal and optimized dimers is zero. Figure 5 presents the strong linear relationship between [?]q and $E^{(2)}$ with the exception of $7 \mathrm{~b}$. Dimer $7 \mathrm{~b}$ forms more I. . . H halogen bonds compared to other dimers. The linear relationship between [?]q and $E^{(2)}$ indicates that charge transfer is an important factor in the noncovalent bonds seen in crystal systems.

TABLE 5 is near here

FIGURE 5 is near here

\subsubsection{Topological Properties}

The topological and energetic properties at the BCPs of the interactions between the two molecules in the six dimers were analyzed by comparing the following parameters; the electron density $\rho$ (b), the Laplacian of electron density $[?]^{2} \rho(\mathrm{b})$, the kinetic electron energy density $\mathrm{G}_{\mathrm{b}}$, the potential electron energy density $V_{b}$, the sum of $G_{b}$ and $V_{b}\left(H_{b}\right)$ and the ratio of $G_{b}$ to $V_{b}\left(-G_{b} / V_{b}\right)$. The results are collected in Table 6. A positive value of $[?]^{2} \rho$ (b) implies an interaction between closed shell complexes; ionic interaction, van der Waals force, or hydrogen bonding, while a negative value of $[?]^{2} \rho$ (b) indicates a shared interaction as in a covalent bond ${ }^{48}$. Rozas's study ${ }^{49}$ concluded that $[?]^{2} \rho(\mathrm{b})$ and $\mathrm{H}_{\mathrm{b}}$ may be useful in characterization of the strength of interactions. This means that for weak A...B interactions, where $[?]^{2} \rho(b)>0$ and $\mathrm{H}_{\mathrm{b}}>$ 0 , the interactions are mainly electrostatic; for medium strength interactions, where[?] ${ }^{2} \rho(\mathrm{b})>0$ and $\mathrm{H}_{\mathrm{b}}$ $<0$, the interactions are partly covalent in nature, while strong interactions show $[?]^{2} \rho(\mathrm{b})<0$ and $\mathrm{H}_{\mathrm{b}}<$ 0 , these are characteristically covalent. Measures of the covalency in noncovalent interactions include the kinetic electron energy density Gc (positive), the potential electron energy density $\mathrm{Vc}$ (negative), and the ratio-Gc/Vc. Values of $-\mathrm{Gc} / \mathrm{Vc}>1$ generally indicate a noncovalent interaction, whereas when $-\mathrm{Gc} / \mathrm{Vc}$ is $<1$, the interaction is covalent in nature. For the dimers investigated here (in Table 6 ), all $[?]^{2} \rho$ (b) values are positive, the $\mathrm{Hc}$ values are positive, and the $-\mathrm{Gc} / \mathrm{Vc}$ values are greater than 1 . This means that these interactions belong to weak interactions of an electrostatic nature.

\section{TABLE 6 is near here}

The electron density, $\rho(\mathrm{b})$, at the bond critical point is used to describe the strength of a bond, where the larger the value of $\rho$ (b), the stronger the bond. In dimer 2 (see Figure 5) the intermolecular halogen bond of I1 .. I18 is the strongest with $\rho(\mathrm{b})=0.008$ au. In dimer 3 the halogen bonds of $\mathrm{I} 2 \ldots \mathrm{C} 27(\pi)$ and $\mathrm{I} 17 \ldots \mathrm{C} 12(\pi))$ were strongest; their $\rho(\mathrm{b})$ was 0.007 au. In dimer 5 the $\mathrm{I} 2 \ldots \mathrm{O} 25$ bond was the strongest with a $\rho(\mathrm{b})$ value of $0.01 \mathrm{au}$. In dimer 6 the I1.. C33( $\pi$ ) interaction was the strongest and its $\rho$ (b) was 0.008 au. In dimer $7 \mathrm{~b}$ the $\mathrm{I} 35 \ldots \mathrm{C} 16(\pi)$ bond was the strongest and its $\rho(\mathrm{b})$ was $0.009 \mathrm{au}$. In dimer 8 the 
I12.. O O33 was the strongest and its $\rho$ (b) was $0.015 \mathrm{au}$. The results concluded from the topological measures of interaction properties are coincident with the experimental results reported in Table 3.

FIGURE 6 is near here

Figure 6 presents the regions of electronic concentration and depletion along each bond in the six dimers; both the contour maps (left) and relief maps (right) of $[?]^{2} \rho$ (b) are shown. The blue points denote BCPs. The blue lines denote positive Laplacian of electron density, which indicate interactions of an ionic character (e.g. van der Waals or intermolecular interactions), and the red lines denote negative Laplacian of electron density which indicate covalent bonds. The corresponding values of the Laplacian of electron density are listed in Table 6. Relief maps provide an intuitive view of the Laplacian of electron density, the curves above the plane show the positive Laplacian of electron density, and the curves below the plane show the negative Laplacian of electron density.

FIGURE 7 is near here

Figure 7 shows the bond lengths of halogen bonds (e.g. I. . I, I. . O, I. . C $(\pi)$ in Table 3 ) and their relationship with electron densities $\rho$ (b). For the halogen bonds, the lower the electron density, the longer the bond length. Therefore, the electron density might serve as a rough measure to estimate the strength of halogen bonding interactions.

\section{4 | CONCLUSION}

In this study, $a b$ initio and QTAIM studies were performed to explore the nature of halogen bonds and some other noncovalent bonds in a series of crystal structure geometries of 1,2-diiodoolefins. Theab initio calculations were carried out at the B3LYP-D3/6-311++G(d,p) and B3LYP-D3/def2-TZVP levels of theory for both crystal and optimized monomers and dimers. Firstly, the calculation results show deviations between the two levels of theory to be quite small. Secondly, the computational values for optimized structures are close to the values for crystal structures.

The reported results provide important information concerning the physical chemistry of these materials. In particular, the crystal geometrical architecture and intermolecular bonding properties were shown to be reproducible with the calculations. The interaction energy and electron density appear to be appropriate tools to judge the stabilities of the crystal structures. Quantification of the noncovalent bonding energy between the molecules in dimers was evaluated both on the crystal and optimized structures, and the interaction energies were within $11.67 \mathrm{~kJ} \cdot \mathrm{mol}^{-1}$ and $44.55 \mathrm{~kJ} \cdot \mathrm{mol}^{-1}$ with B3LYP-D3/6-311++G(d,p). The intermolecular interactions responsible for the formation of the dimers are weak-to-moderate in strength, these interactions were clearly of enough local significance to guide the solid state crystallization process. Moreover, for the halogen bonds of type I...I, I...O and I...C $(\pi)$, there is a strong linear relationship between the electron densities $\rho$ (b) and the bond lengths. This confirms the relationships between electron density and the stability of halogen bonds.

\section{CONFLICT OF INTEREST}

There are no conflicts to declare.

\section{ACKNOWLEDGEMENTS}

This work was supported by the Fundamental Research Funds for the Central Universities (Grant No. lzujbky-2019-cd05), the National Natural Science Foundation of China (No. 81973786), and the Science Foundation of Guangxi (AA17204096, AD16380076).

\section{REFERENCES}

1. H. P. Shing, Future Med. Chem. 2017, 9 , 637-640.

2. S. H. Jungbauer, D. Bulfield, F. Kniep, C. W. Lehmann, E. Herdtweck and S. M. Huber, J. Am. Chem. Soc. 2014, 136, 16740-16743. 3. F. G. L. Parlane, C. Mustoe, C. W. Kellett, S. J. Simon, W. B. Swords, 
G. J. Meyer, P. Kennepohl and C. P. Berlinguette, Nat. Commun. 2017, 8, 1761-1768. 4. P. Metrangolo, G. Resnati and T. Pilati, Struct. Bond 2008,126, 105-136. 5. K. Raatikainen and K. Rissanen, Chem. Sci. 2012, 3, 1235-1239. 6. G. R. Desiraju, P. S. Ho, L. Kloo, A. C. Legon, R. Marquardt, P. Metrangolo, P. Politzer, G. Resnati and K. Rissanen, Pure Appl. Chem. 2013, 85, 1711-1713. 7. G. Cavallo, P. Metrangolo, R. Milani, T. Pilati, A. Priimagi, G. Resnati and G. Terraneo, Chem. Rev. 2016, 116, 2478-2601. 8. M. Erdelyi, Chem. Soc. Rev. 2012, 41, 3547-3557. 9. E. Parisini, P. Metrangolo, T. Pilati, G. Resnati and G. Terraneo,Chem. Soc. Rev. 2011, 40, 2267-2278.

10. A. C. Carlsson, J. Gräfenstein, A. Budnjo, J. L. Laurila, J. Bergquist, A. Karim, R. Kleinmaier, U. Brath and M. Erdélyi, J. Am. Chem. Soc. 2012, 134, 5706-5715.

11. L. Shuman, X. Tianlv, T. van Mourik, H. Früchtl, S. R. Kirk and S. Jenkins, Molecules 2019, 24 , 2875-2886.

12. R. B. Walsh, C. W. Padgett and P. Metrangolo, Cryst. Growth Des. 2001, 1, 165-175.

13. R. B. K. Siram, D. P. Karothu, T. N. G. Row and S. Patil,Cryst. Growth Des. 2013, 13 , 1045-1049.

14. S. J. Grabowski, Phys. Chem. Chem. Phys. 2013, 15 , 7249-7259.

15. P. Metrangolo and G. Resnati, Science 2008, 321, 918-919.

16. D. Cinčić, T. Friščić and W. Jones, CystEngComm . 2011,13, 3224-3231.

17. P. Deepa, B. V. Pandiyan, P. Kolandaivel and P. Hobza, Phys. Chem. Chem. Phys. 2014, 16 , 2038-2047.

18. L. Koskinen, P. Hirva, E. Kalenius, S. Jääskeläinen, K. Rissanen and M. Haukka, CrystEngComm. 2015, $17,1231-1236$.

19. R. F. W. Bader, Chem. Rev. 1991, 91 , 893-928.

20. T. Clark, M. Hennemann, J. S. Murray and P. Politzer, J. Mol. Model 2007, 13 , 291-296.

21. P. Politzer, P. Lane, M. C. Concha, Y. Ma and J. S. Murray, J. Mol. Model 2007, 13 , 305-311.

22. Y. Zeng, X. Zhang, X. Li, S. Zheng and L. Meng, Int. J. Quan. Chem. 2010, $111,3725-3740$.

23. X. Zhang, Y. Zeng, X. Li, L. Meng and S. Zheng, Struct. Chem.2011, 22, 567-576.

24. S. J. Grabowski, J. Phys. Chem. A 2012, 116 , 1838-1845.

25. A. Bauzá, S. K. Seth and A. Frontera, J. Comput. Chem. 2018,39 , 458-463.

26. Y. Roselló, M. Benito, E. Molins, M. Barceló-Oliver and A. Frontera, Crystals 2019, 9 , 224-233.

27. K. Wzgarda-Raj, A. J. Rybarczyk-Pirek, S. Wojtulewski and M. Palusiak, Acta Crystallogr. C. 2020, 76 , 170-176.

28. M. Domagała, A. Lutynska and M. Palusiak, J. Phys. Chem. A2018, 122 , 5484-5492.

29. C. Hettstedt, P. Mayer and K. Karaghiosoff, New J. Chem.2015, 39 , 8522-8533.

30. F. Weigend and R. Ahlrichs, Phys. Chem. Chem. Phys. 2005,7, 3297-3305.

31. S. Grimme, J. Antony, S. Ehrlich and H. Krieg, J. Chem. Phys.2010, 132, 154104-154123.

32. S. Grimme, S. Ehilich and L. Goerigk, J. Comput. Chem. 2011,32 , 1456-1465.

33. M. J. Frisch, G. W. Truchs, H. B. Schlegel, G. E. Scuseria, M. A. Robb, J. R. Cheeseman, G. Schmani, V. Barone, B. Mennucci, G. A. Petersson, H. Nakatsuji, M. Caricato, X. Li, H. P. Hratchian, A. F. Izmaylov, J. Bloino, G. Zheng, J. L. Sonnenberg, M. Hada, M. Ehara, K. Toyota, R. Fukuda, J. Hasegawa, M. Ishida, T. Nakajima, Y. Honda, O. Kitao, H. Nakai, T. Vreven, J. J. A. Montgomery, J. E. Peralta, F. 
Ogliaro, M. Bearpark, J. J. Heyd, E. Brothers, K. N. Kudin, V. N. Staroverov, R. Kobayashi, J. Normand, K. Raghavachari, A. Rendell, J. C. Burant, S. S. Iyengar, J. Tomasi, M. Cossi, N. Rega, N. J. Millam, M. Klene, J. E. Knox, J. B. Cross, V. Bakken, C. Adamo, J. Jaramillo, R. Gomperts, R. E. Stratmann, O. Yazyev, A. J. Austin, R. Cammi, C. Pomelli, J. W. Ochterski, R. L. Martin, K. Morokuma, V. G. Zakrzewski, G. A. Voth, P. Salvador, J. J. Dannenberg, S. Dapprich, A. D. Daniels, Ö. Farkas, J. B. Foresman, J. V. Ortiz, J. Cioslowski and D. J. Fox, Gaussian 09,Revision E.01. Gaussian 09, Revision E.01 , Gaussian, Inc., Wallingford CT. Wallingford CT, 2015.

34. H. Zhao, J. Chang and L. Du, Comput. Theor. Chem. 2016,1084, 126-132.

35. Q. Zhang and L. Du, Comput. Theor. Chem. 2016, 1078 , 123-128.

36. S. Tang, H. Zhao and L. Du, RSC Adv. 2016, 6 , 91233-91242.

37. A. Bauza, D. Quinonero, P. M. Deya and A. Frontera,CrystEngComm. 2013, 15, 3137-3144.

38. M. Kolař, J. Hostaš and P. Hobza, Phys. Chem. Chem. Phys.2014, 16 , 9987-9996.

39. A. Bauzá, R. Ramis and A. Frontera, Comput. Theor. Chem.2014, 1038 , 67-70.

40. S. F. Boys and F. Bernardi, Mol. Phys. 1970, 19 , 553-566.

41. R. F. W. Bader, M. T. Carroll, J. R. Cheeseman and C. Cheng, J. Am. Chem. Soc. 1987, 109, 7968-7979.

42. E. D. Glendening, J. K. A. E. Reed, J. E. Carpenter and F. Weinhold, NBO 3.1, QCPE Bull, 1990, 10 , 58.

43. T. A. Keith, AIMAll, 15.09.27, TK Gristmill Software, 2011.

44. F. Zordan, L. Brammer and P. Sherwood, J. Am. Chem. Soc . 2005, 127 , 5979-5989.

45. M. Domagała, P. Matczak and M. Palusiak, Comput. Theor. Chem.2012, 998 , 26-33.

46. M. Domagała and M. Palusiak, Comput. Theor. Chem. 2014,1027, 173-178.

47. M. Domagała, A. Lutyńska and M. Palusiak, Int. J. Quantum Chem. 2017, 117 , e25348-e25356.

48. P. L. A. Popelier, Atoms in molecules: an introduction.Prentice Hall, Pearson Education Limited, New York, 2000.

49. I. Rozas, I. Alkorta and J. Elguero, J. Am. Chem. Soc. 2000,122, 11154-11161.

\section{Hosted file}

Figures.docx available at https://authorea.com/users/347215/articles/473003-halogen-bondsand-other-noncovalent-interactions-in-the-crystal-structures-of-1-2-diiodo-alkenes-anab-initio-and-qtaim-study

\section{Hosted file}

Tables.docx available at https://authorea.com/users/347215/articles/473003-halogen-bondsand-other-noncovalent-interactions-in-the-crystal-structures-of-1-2-diiodo-alkenes-anab-initio-and-qtaim-study 